\title{
Active Torque Harmonic Compensation for Wind Turbine Drive Trains
}

\author{
L. Peretti* \\ *ABB Corporate Research, Power Technologies Department, Västerås, Sweden, email: luca.peretti@se.abb.com
}

Keywords: AC drives, wind turbines, torque harmonics, active compensation

\begin{abstract}
This paper presents a torque harmonic compensation algorithm for wind turbine applications, where the presence of a converter managing the energy conversion can be also exploited for active compensation purposes. A general scenario is described first. The algorithm is then described with its mathematical background. A modification of the algorithm is proposed in order to overcome the problem of unknown phase shift between the variables to compensate and those steering the compensation. Simulations and experimental results prove the effectiveness of the solution.
\end{abstract}

\section{Introduction}

Torque harmonic compensation in wind turbine drive trains is a topic that recently gained interest in the research scenario. As power generation cost issues are becoming large driving factors in wind energy applications, the damping of mechanical vibrations in the turbine drive train for a reduction of maintenance and an increase of the lifetime of the mechanical components is an appealing target [1].

The topic is not novel, having been investigated for many other applications, as for example servo or industrial ones (see the references contained in [1] for further details). The case of the wind application is attractive because of the composition of disturbances, which encompasses mechanics of the gearbox ([1]), aerodynamics (like wind shear and tower shadow effects, [2]), electrics ([3], [4]), electronics ([5]) and others. A multidisciplinary approach is needed to understand the vibration phenomena as a whole.

The converter driving the generator is potentially able, to certain extent, to perform the vibration reduction for any disturbance visible on the rotating axis of the drive train shaft. The vibration reduction capability should be considered as a software feature completing the main generator control, as a function that can be enabled or disabled upon request.

This paper describes a software feature that performs torque harmonic compensation in wind turbine drive train systems by exploiting the frequency converter. Its theoretical background is discussed in Sect. 2, with simulations presented in Sect. 3. Experiments on a small-scale laboratory prototype are included in Sect. 4. Conclusive remarks are given in Sect. 5 , followed by acknowledgements and references.

\section{Theoretical background}

\subsection{The proposed schematic}

A general scheme of the proposed compensator is shown in Figure 1. The software feature is called "multiple-frequencies compensator" (MFC). The MFC is implemented as an additional function of the generator control core program. Figure 2 expands the MFC in its internal blocks, showing a frequency identifier and a group of frequency compensators.

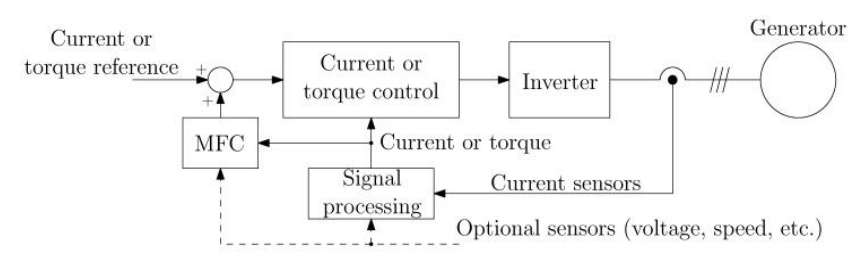

Figure 1: General scheme of the MFC as a software feature.

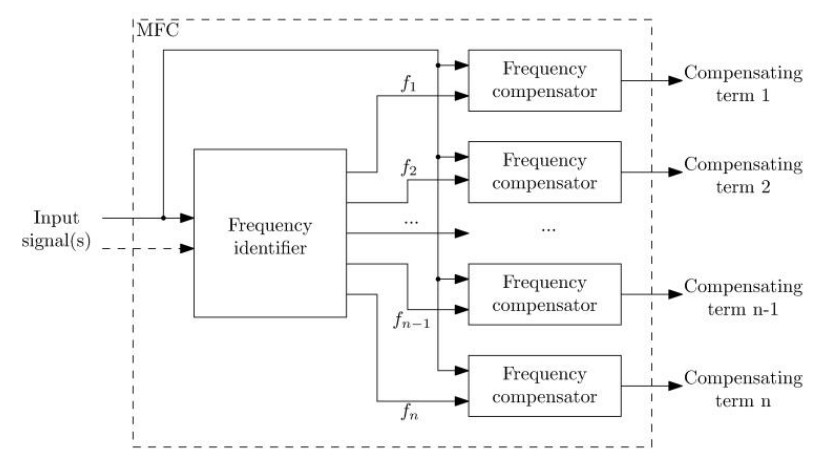

Figure 2: Internal structure of the MFC.

The frequency identifier estimates the disturbance frequencies from the input signals (current, torque, speed, etc.). The output of the harmonic identifier is a group of frequencies $f_{1}, \ldots, f_{n}$, each of them requiring a compensation action.

A single frequency compensator performs the action for one of the identified frequencies, on a signal requiring the damping action. The nature of its output varies depending on the nature of the generator main control. A current or a torque reference could be generated, respectively for a field-oriented control (FOC) or a direct-torque control (DTC). 
The schematic of the single harmonic compensator with no phase shift (see similar schemes in [6], [7]) is reported in Fig. 3. Its behaviour is briefly described:

- The high-pass filters remove the mean value of the inputs $g_{\alpha}$ and $g_{\beta}$, obtaining two AC values $g_{\alpha, h p}$ and $g_{\beta, h p}$;

- the transformation in a reference frame $x y$ rotating with the previously identified frequency obtains meaningful DC values $g_{x, h p}$ and $g_{y, h p}$ at the harmonic of interest;

- the low-pass filters remove any frequency excluding the meaningful DC values, keeping only $g_{x, b p}$ and $g_{y, b p}$;

- the regulators $\operatorname{Reg}_{x}$ and $\operatorname{Reg} g_{y}$ reject the disturbance by force the magnitude of the input at zero, generating two compensating signals $c_{x, b p}$ and $c_{y, b p}$;

- the transformation back to the stationary $\alpha \beta$ reference frame generates the signals $c_{\alpha, b p}$ and $c_{\beta, b p}$ used as current or torque references in the generator control loop.

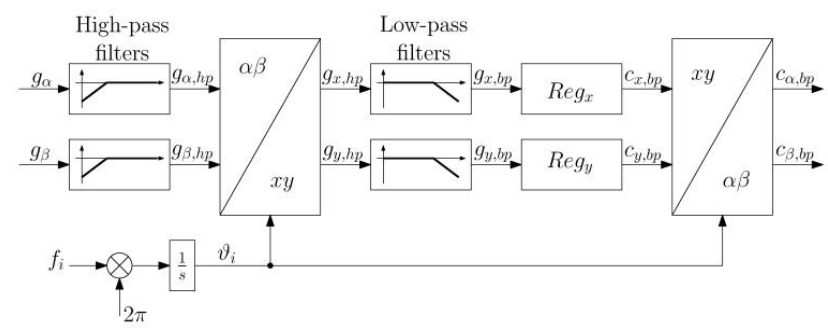

Figure 3: Scheme of the proposed single harmonic compensator without phase shifter.

Sect. 2.2 describes the mathematical background of the compensator. Because of its generality, the vector input case is treated. The scalar input case is then obtained as a special case of the vector input one.

\subsection{The vector input case}

In this case, a vector input with two components $\boldsymbol{g}_{\boldsymbol{\alpha}}$ and $\boldsymbol{g}_{\boldsymbol{\beta}}$ is sent to the compensator. The components contain both DC values and sinusoidal values with an angular frequency $\boldsymbol{\omega}_{\boldsymbol{i}}$ :

$$
\begin{aligned}
& g_{\alpha}=G_{\alpha, \text { offs }}+G_{\alpha} \cos \left(\omega_{i} t\right) \\
& g_{\beta}=G_{\beta, o f f s}+G_{\beta} \sin \left(\omega_{i} t\right)
\end{aligned}
$$

According to the scheme of Figure 3, the (1) are high-pass filtered obtaining:

$$
\begin{aligned}
& g_{\alpha, h p}=G_{\alpha} \cos \left(\omega_{i} t\right) \\
& g_{\beta, h p}=G_{\beta} \sin \left(\omega_{i} t\right)
\end{aligned}
$$

The compensator then performs a rotation using an angle $\vartheta_{i}=\omega_{i} t+\varphi$. Note that $\vartheta_{i}$ is the integral of the angular frequency needing compensation, without assumptions on its phase. The input components in the $x y$ reference frame are:

$\left[\begin{array}{l}g_{x, h p} \\ g_{y, h p}\end{array}\right]=T_{\alpha \beta / x y}\left[\begin{array}{l}g_{\alpha, h p} \\ g_{\beta, h p}\end{array}\right]=\left[\begin{array}{cc}\cos \left(\vartheta_{i}\right) & \sin \left(\vartheta_{i}\right) \\ -\sin \left(\vartheta_{i}\right) & \cos \left(\vartheta_{i}\right)\end{array}\right]\left[\begin{array}{l}g_{\alpha, h p} \\ g_{\beta, h p}\end{array}\right]$
Substituting the (2) in the (3), one obtains:

$$
\begin{aligned}
& g_{x, h p}=\frac{G_{\alpha}+G_{\beta}}{2} \cos (\varphi)+\frac{G_{\alpha}-G_{\beta}}{2} \cos \left(2 \omega_{i} t+\varphi\right) \\
& g_{y, h p}=-\frac{G_{\alpha}+G_{\beta}}{2} \sin (\varphi)-\frac{G_{\alpha}-G_{\beta}}{2} \sin \left(2 \omega_{i} t+\varphi\right)
\end{aligned}
$$

These components are low-pass filtered in order to keep their DC value. Assuming that the transient of the low-pass filter is concluded, the components become:

$$
\begin{aligned}
& g_{x, h p}=\frac{G_{\alpha}+G_{\beta}}{2} \cos (\varphi) \\
& g_{y, h p}=-\frac{G_{\alpha}+G_{\beta}}{2} \sin (\varphi)
\end{aligned}
$$

The low-pass-filtered components in (5) carry the information on the input magnitude, which should be controlled to zero. A regulator is therefore employed (see Figure 3). With a simple proportional-integral controller with proportional gain $K_{p}$ and integral gain $K_{i}$, it follows:

$$
\begin{aligned}
& c_{x, b p}=K_{p} g_{x, b p}+K_{i} \int_{0}^{t} g_{x, b p} d t \\
& c_{y, b p}=K_{p} g_{y, b p}+K_{i} \int_{0}^{t} g_{y, b p} d t
\end{aligned}
$$

The output of the regulators is transformed back in the $\alpha \beta$ reference frame, leading to:

$$
\begin{aligned}
& c_{\alpha, b p}=\left(K_{p}+K_{i} t\right) \frac{G_{\alpha}+G_{\beta}}{2} \cos \left(\omega_{i} t\right) \\
& c_{\beta, b p}=\left(K_{p}+K_{i} t\right) \frac{G_{\alpha}+G_{\beta}}{2} \sin \left(\omega_{i} t\right)
\end{aligned}
$$

The result in (7) shows that the output of the compensator is a signal in phase with the input signal, with increasing output due to the integral action of the regulator. More importantly, the phase of the transformation does not have any impact on the result of the compensation. The compensator works well also with scalar signals (for example, with $G_{\alpha} \neq 0$ and $G_{\beta}=0$ ). In this case, the output signal on the $\alpha$ axis will be in phase with the input, while the output signal on the $\beta$ axis can be simply discarded. This consideration opens the possibility of exploiting the same compensator scheme for scalar signals like speed, acceleration, torque, vibration on one axis, etc.

\subsection{The compensator with phase shifter}

The (7) shows that the output signal is in phase with the input signal. This may contrast with situations in which, for example, the input is a speed and the compensation is a torque reference. The transfer function from the torque to the speed is usually a low-pass filter (with or without resonances), therefore a phase shift between torque and speed is expected. It is not possible to obtain a phase shift with the scheme of Fig. 3. In order to achieve full compensation capabilities, a modification of Fig. 3 is introduced as shown in Fig. 4. 
The modification consists of a block named "phase shifter", which multiplies the high-pass-filtered input signal and the compensating signal, both on the $\alpha$ axis. The multiplication is low-pass filtered extracting its mean value, and sent to an integrator to generate a phase lead for the second transformation. The modification suits the cases where the phase shift between the input and the output signals is unknown.

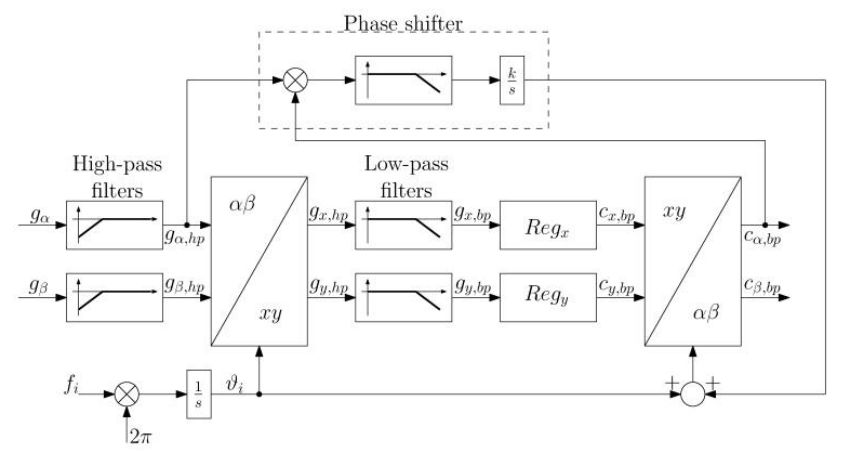

Figure 4: Scheme of the proposed single harmonic compensator with the phase shifter.

The effect of the phase shifter is to advance the compensating signal of a quantity related to the average value of the multiplication. The phase advancement is stabilised when the input signal reaches zero amplitude.

An issue arises when the compensating signal needs a phase shift between $\pi$ and $2 \pi$. Such issue is taken into account with an easy fix: prior the activation of the phase shifter, the simple compensator is run without integral action. The compensation signal is added to the reference signal and the effect of the compensation is observed. If the harmonic has been slightly reduced, then the sign is correct and there is no required change. On the contrary, if the harmonic has been slightly increased, the sign of the compensating output must be changed. The phase shifter does not need to be changed.

\subsection{Tuning of the high-pass filter}

It is recommended to use high-pass filters with a stop-band region as close to $\omega_{i}$ as possible, since this leads to faster transients. A large stop-band region is also beneficial for the removal of lower-order harmonics which should not be compensated by the compensator (see Sect. 2.6).

\subsection{Tuning of the low-pass filter}

Let the high-pass-filtered input signal be of the form of (2), with a generic angular frequency $\omega_{g}$ instead of $\omega_{i}$. The $x y$ components, in this case, are equal to:

$$
\begin{aligned}
& g_{x, h p}=\frac{G_{\alpha}+G_{\beta}}{2} \cos \left(\left(\omega_{g}-\omega_{i}\right) t-\varphi\right)+\frac{G_{\alpha}-G_{\beta}}{2} \cos \left(\left(\omega_{g}+\omega_{i}\right) t+\varphi\right) \\
& g_{y, h p}=\frac{G_{\alpha}+G_{\beta}}{2} \sin \left(\left(\omega_{g}-\omega_{i}\right) t-\varphi\right)-\frac{G_{\alpha}-G_{\beta}}{2} \sin \left(\left(\omega_{g}+\omega_{i}\right) t+\varphi\right)
\end{aligned}
$$

The (8) shows that a generic harmonic is split in two harmonics in the rotating reference frame $x y$, and falls back to (4) when $\omega_{g}=\omega_{i}$. When the input signals in $\alpha \beta$ are composed by two or more harmonics, the results of both (4) and (8) must be combined. In order to maintain only the meaningful signal of interest (that is, the DC part of the (4)), it is suggested to tune the low-pass filter in order to remove all harmonics higher than $\omega_{g}=\omega_{i}$ in the $x y$ reference frame. Combined with the high-pass filter action (whose cut-off frequency is proposed to be closer to $\omega_{i}$ ), the effect of spurious harmonics in the $x y$ reference frame is minimised, although not completely eliminated (for $\omega_{g}$ slightly larger than $\omega_{i}$, a spurious harmonic at $\omega_{g}-\omega_{i}$ appears, but it is believed that the system can tolerate it).

\subsection{Tuning of the regulators}

The tuning of the regulators in Figure 4 is a task that involves many different aspects of the system. Input signals with different nature can be used, and the analysis of possible combinations of control loop diagrams is not straightforward. An important consideration is that the integral part of the regulators must be tuned carefully with respect to the phase shifter gain, in order to avoid unwanted dynamics.

\section{Simulation results}

Simulations were performed in order to test the compensation within a wind turbine system. As a simulation case, a directdrive synchronous generator system described in [8] was used, with two mechanical resonances located respectively at $9,29 \mathrm{~Hz}$ and $164,58 \mathrm{~Hz}$. The turbine model was the one described in [9]. The generator was rotating at rated speed (around $15 \mathrm{rpm}$ ) with a partial load of 1,5 MW (out of 4 $\mathrm{MW}$ ), and an injected disturbance with frequency equal to the first mechanical resonant frequency. The disturbance amplitude was $4 \%$ of the nominal torque of the generator (see [8] for the generator data), injected on the generator side. The compensator used the speed as input signal, and produced a torque compensation signal used by the generator control loop. The reason for the 1,5-MW partial generation was that the turbine is not supposed to work always at rated load and, besides, a margin for a torque compensation is needed.

The results are shown from Figure 5 to Figure 12. The red dashed vertical line shows the time instant of the compensation activation. While the turbine rotor side is not very much affected by the injected disturbance (see Figure 5, Figure 6, Figure 7 and Figure 8), the generator side shows an increased harmonic content before the activation of the MFC (see generator speed, Figure 10). The MFC activation at $5 \mathrm{~s}$ cleans the harmonic from the speed, giving even better compensation when the phase shifter is activated at $10 \mathrm{~s}$ (Figure 11). The generator torque becomes oscillatory (see Figure 9), as well as the DC-bus voltage (Figure 12), which is affected by an oscillation of approximately $50 \mathrm{~V}$. 


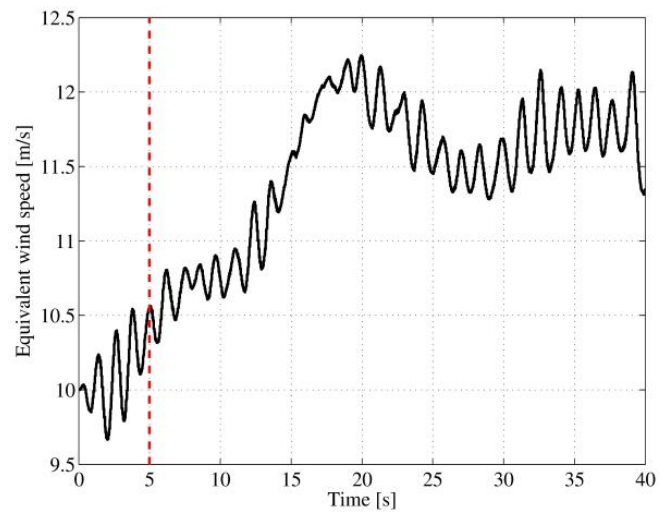

Figure 5: Simulated equivalent wind speed.

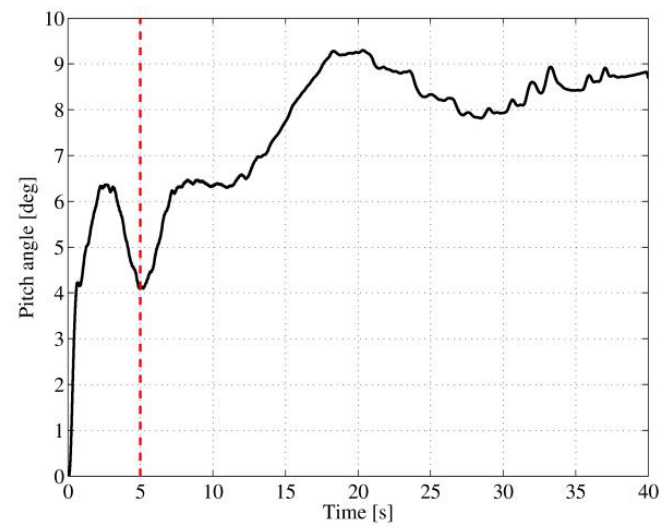

Figure 6: Simulated pitch angle.

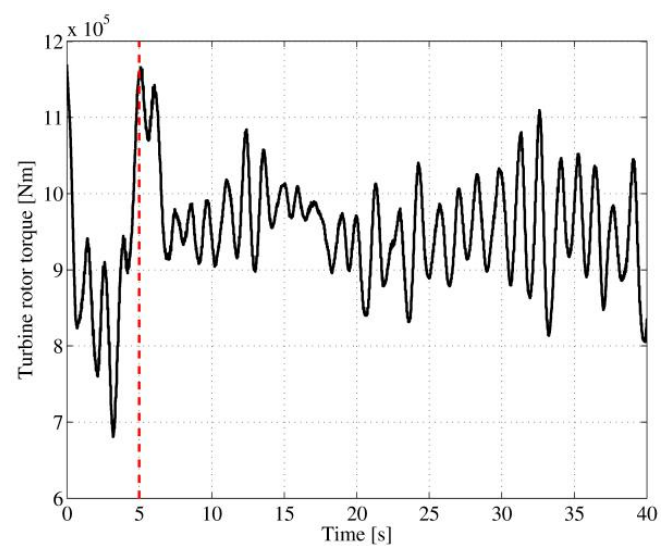

Figure 7: Simulated tubine rotor torque.

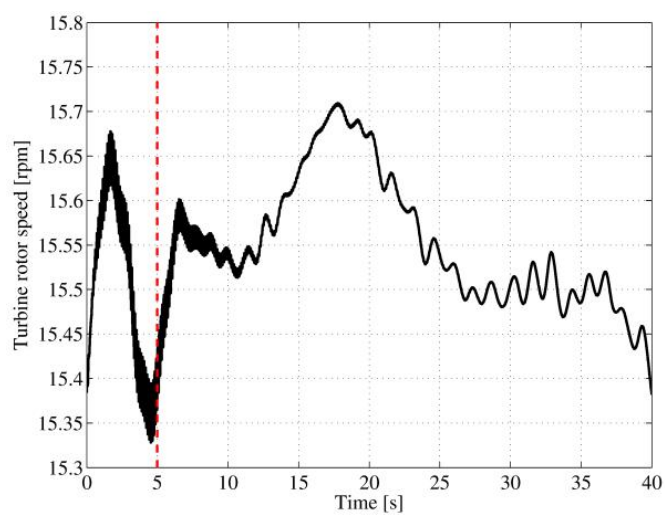

Figure 8: Simulated turbine rotor speed.

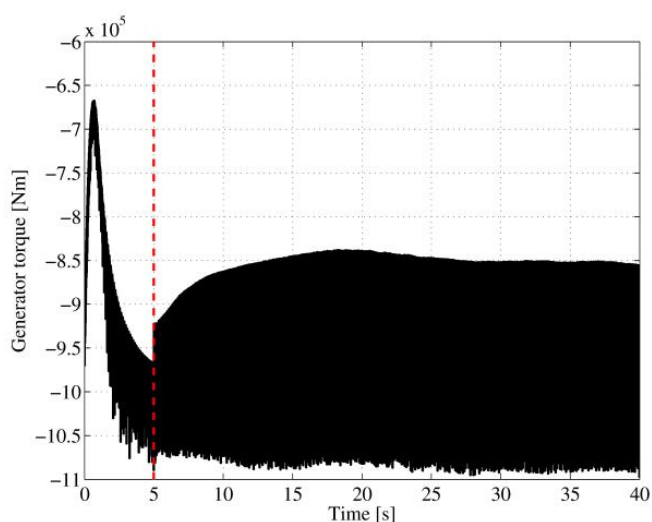

Figure 9: Simulated generator torque.

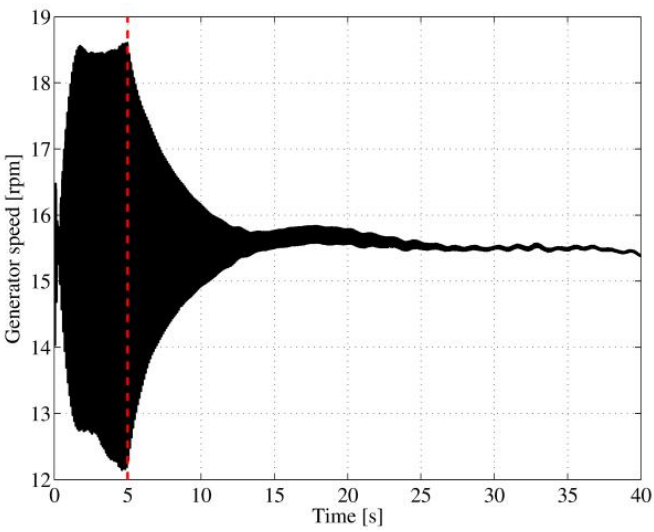

Figure 10: Simulated generator speed.

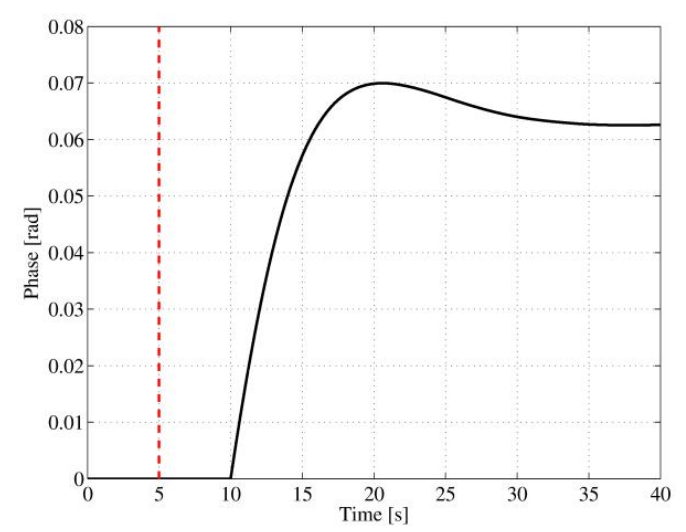

Figure 11: Simulated phase compensation.

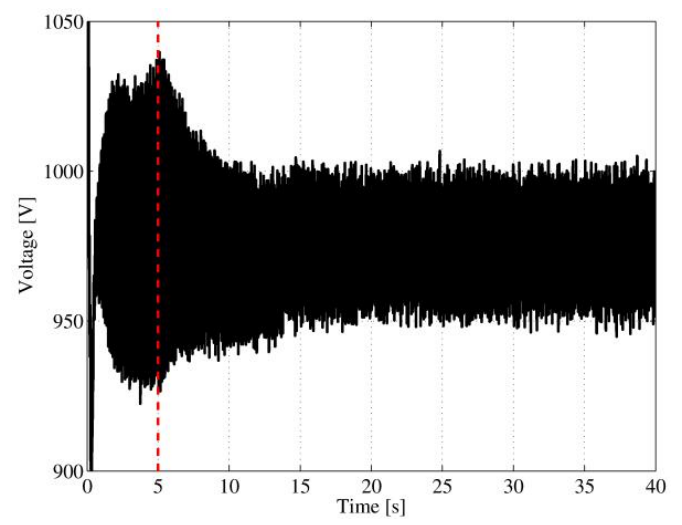

Figure 12: Simulated DC-bus voltage. 


\section{Experimental results}

Experimental tests were performed on the small-scale wind turbine emulator described in [10], composed by two 2-kW permanent-magnet synchronous machines and two $4-\mathrm{kW}$ ABB ACSM1 drives, running at roughly $4 \mathrm{kHz}$ of switching frequency. The detailed description of the experimental setup, as well as the data related to the turbine model and the overall control of the wind turbine emulator, are included in [10].

The tests aimed to evaluate the compensation behaviour in steady-state and transient conditions. An experiment was created by injecting three torque harmonics from the load machine: a $1^{\text {st }}$ harmonic of the mechanical speed (resembling load unbalance), a $3^{\text {rd }}$ harmonic of the mechanical speed (resembling wind shear/tower shadow effects, see [2]), and a constant frequency of $20 \mathrm{~Hz}$ (resembling a resonant frequency in the system). The speed was controlled to be either $100 \mathrm{rpm}$, $200 \mathrm{rpm}$ or $300 \mathrm{rpm}$ by the load machine control.

The THC was activated around $10 \mathrm{~s}$ and released around $87 \mathrm{~s}$ (see Figure 13). During this interval, the torque disturbance from the wind turbine emulator was as in Figure 14, resembling the turbulence of the wind. The compensating action was obtained using the speed as input of the MFC, and generating a torque reference as compensating signal (see Figure 15). A harmonic reduction on the speed is visible, as in the zoomed pictures from Figure 16 to Figure 21.

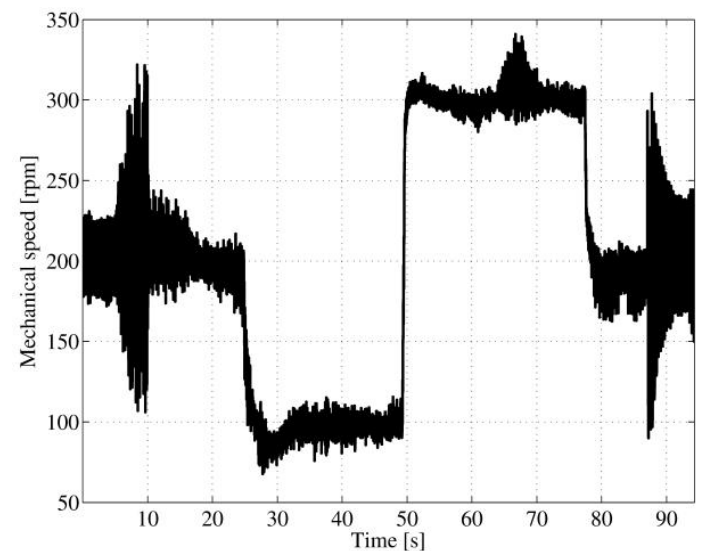

Figure 13: Experimental mechanical speed (complete test).

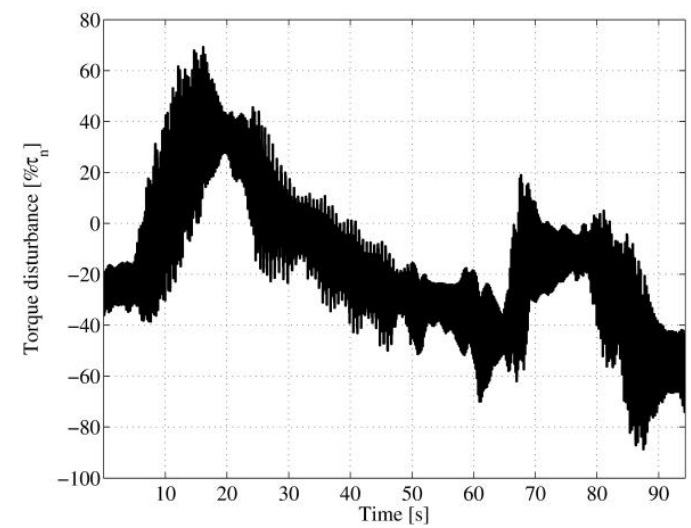

Figure 14: Experimental torque disturbance (complete test).

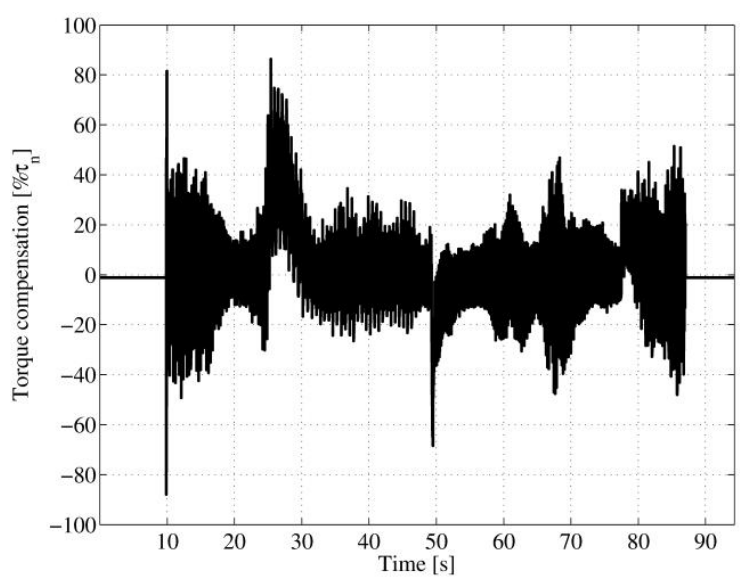

Figure 15: Experimental torque compensation (complete test).

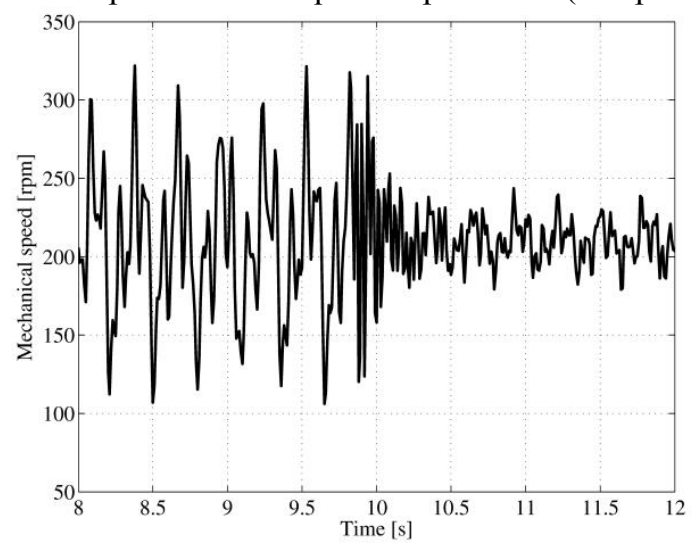

Figure 16: Experimental mechanical speed (zoom 1).

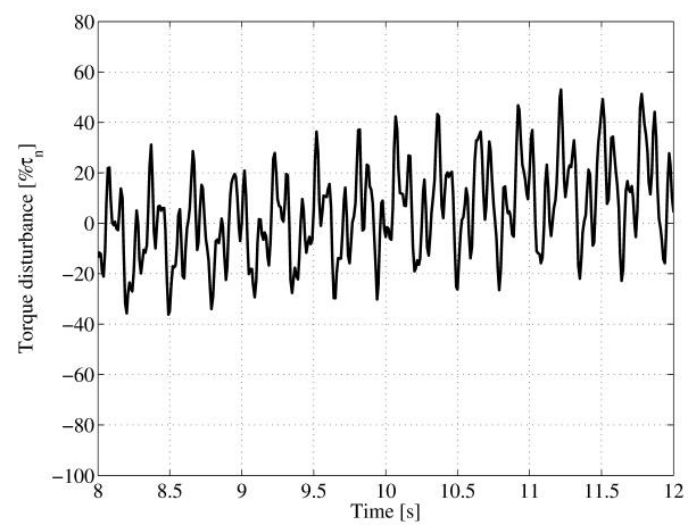

Figure 17: Experimental torque disturbance (zoom 1).

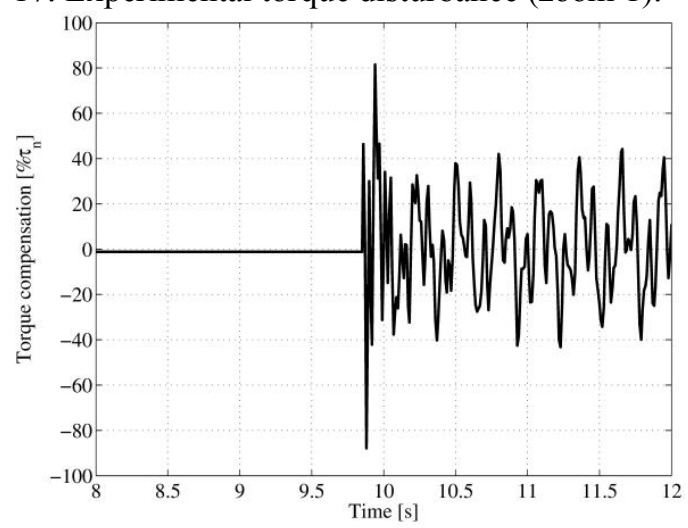

Figure 18: Experimental torque compensation (zoom 1). 


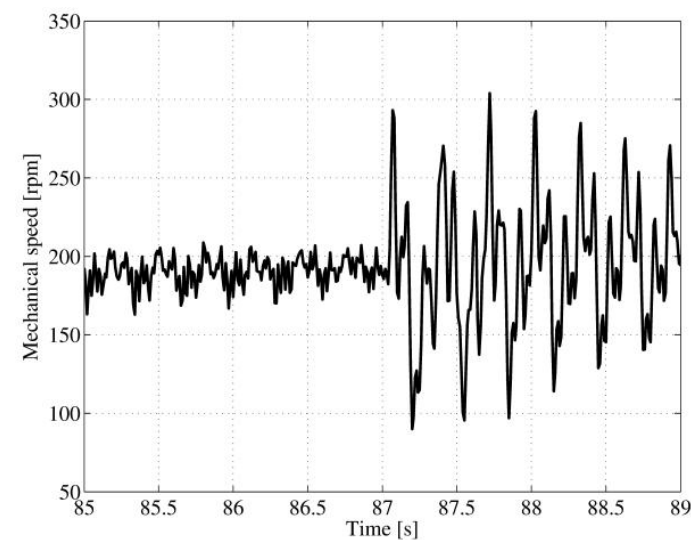

Figure 19: Experimental mechanical speed (zoom 2).

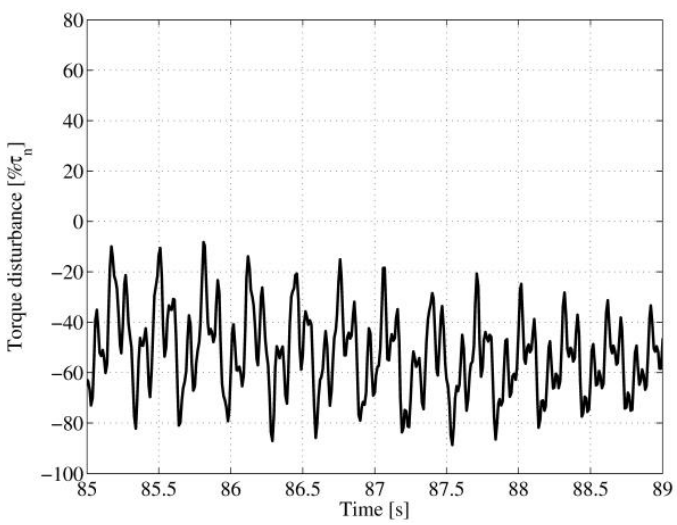

Figure 21: Experimental torque disturbance (zoom 2).

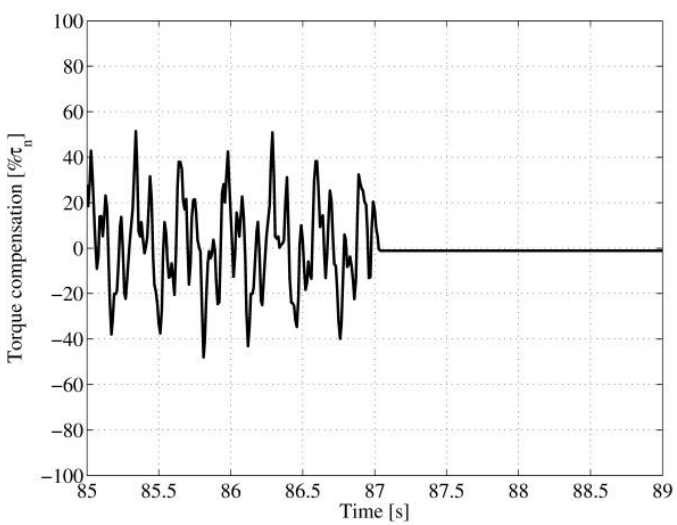

Figure 20: Experimental torque compensation (zoom 2).

\section{Conclusions}

A procedure for the active compensation of torque harmonics in wind turbine applications was analysed, simulated, and experimentally tested on a small-scale laboratory test bench. The compensation is based on the rejection of the harmonic amplitude calculated in a reference frame $\boldsymbol{x} \boldsymbol{y}$ rotating with the frequency of the harmonic. A phase shifter is proposed in order to obtain the required phase shift between the compensation signal and the input signal (in this work, the torque reference and the mechanical speed). The simulated and experimental results showed the validity of the approach, even in presence of more than one harmonic at once.

\section{Acknowledgements}

The Author gratefully acknowledges the help of Mikael Aulanko and Raimo Sakki from ABB Oy, Helsinki, Finland, which was fundamental for the development of this work.

\section{References}

[1] G. Mandic, A. Nasiri, E. Muljadi, F. Oyague, “Active torque control for gearbox load reduction in a variablespeed wind turbine", IEEE Tran. Ind. Appl., vol. 48, no. 6, Nov./Dec. 2012, pp. 2424-2432.

[2] D. S. L. Dolan, P. W. Lehn, "Simulation model of wind turbine $3 p$ torque oscillations due to wind shear and tower shadow", IEEE Trans. En. Conv., vol. 21, no. 3, Sept. 2006, pp. 717-724.

[3] L. Xu, Y. Wang, "Dynamic modeling and control of DFIG-based wind turbines under unbalanced network conditions", IEEE Trans. Pow. Syst., vol. 22, no. 1, Feb. 2007, pp. 314-323.

[4] D. Casadei, F. Filippetti, C. Rossi, A. Stefani, A. Yazidi, and G. A. Capolino, "Diagnostic technique based on rotor modulating signals signature analysis for doubly fed induction machines in wind generator systems," in Conference Record of the 2006 IEEE 41th Industry Application Society Annual Meeting (IAS 2006), Tampa, Florida, Oct. 8-12 2006, pp. 1525-1532.

[5] N. Miyamoto and K. Ohishi, "Online tuning method for current measurement offsets and gain deviations for SPMSM," in Proceedings of the 38th Annual Conference of the IEEE Industrial Electronics Society (IECON 2012), Montréal, Canada, Oct. 25-28 2012, pp. 1940-1945.

[6] J. Wang, D. C. Liu, P. Liu, and J. Zhao, "A new algorithm for random harmonic current detection based on mathematical morphology," in Proceedings of the WRI Global Congress on Intelligent Systems (GCIS 2009), Xiamen, China, May 19-21 2009, pp. 365-369.

[7] M. Li, "An improved harmonic detecting approach for active power filter," in Proceedings of the 3rd International Power Electronics and Motion Control Conference (IPEMC 2000), vol. 3, Beijing, China, Aug. 15-18 2000, pp. 1420-1424.

[8] J. Sopanen, V. Ruuskanen, J. Nerg, J. Pyrhönen, "Dynamic torque analysis of a wind turbine drive train including a direct-driven permanent-magnet generator", IEEE Trans. Ind. Electr., vol. 58, no. 9, Sept. 2011, pp. 3859-3867.

[9] M. Soltani, T. Knudsen, T. Bak, " Modeling and Simulation of Offshore Wind Farms for Farm Level Control", Proceedings of the European Offshore Wind Conference and Exhibition (EOW) 2009, 14-16 September, Stockholm, Sweden, pp. 2052-2061.

[10] L. Peretti, V. Särkimäki, J. Faber, "A wind turbine emulator for generator control algorithm development", IEEE International Conference on Industrial Technology (ICIT), Cape Town, South Africa, Feb. 25-27, 2013, pp. 228-233. 